Seronegative rheumatoid arthritis associated with AIDS

Sir: Acquired immunodeficiency syndrome (AIDS) is a disease of protean manifestations. It is almost certainly caused by a retrovirus: human immunodeficiency virus (HIV). Its major presentations are those of recurrent infection and neoplasia.

Musculoskeletal manifestations have been well reported' and occur in up to $70 \%$ of patients.

Arthritis is less common but certainly does occur. Reiter's syndrome and reactive arthritis are the most commonly associated. ${ }^{12}$ Infectious arthritis due to organisms such as cryptococcus and mycobacteria has been reported.

Psoriatic arthritis occurs. An AIDS associated arthritis has also been described.

Rheumatoid arthritis like disease has rarely been reported as coexisting with AIDS. The patient presented here had criteria for classical seronegative rheumatoid arthritis. His case is presented to alert clinicians to this association and to report that AIDS does not exclude rheumatoid arthritis.

The patient, a white homosexual man, aged 28 , presented in January 1986 with pneumonia. He was admitted to hospital and recovered uneventfully from a diagnosed Streptococcus pneumoniae pneumonia. At that time he was noted to have cervical adenopathy and a packed cell volume of 0.34 with a normochromic normocytic blood smear. In April 1986 he developed swelling and pain in his feet and then his knees, with generalised arthralgia. He was evaluated for this in May 86 and was found to be HLA-B27 positive with no sacroiliitis or other stigmata of Reiter's syndrome. His haemoglobin was $119 \mathrm{~g} / \mathrm{l}$; packed cell volume 0.36 ; white cell count $11.5 \times 10^{9} / 1$ with mild neutropenia; and erythrocyte sedimentation rate $51 \mathrm{~mm} / \mathrm{h}$. Other pertinent results included negative LE cells, antinuclear antibody titre positive at $1 / 40$ with a homogeneous pattern, antistreptolysin $\mathrm{O}$ streptozyme 1:100 STZ units. Complement concentrations were normal. Synovial fluid analysis of the knee showed an inflammatory exudate of $9.5 \times 10^{9} / 1$ white blood cells with $78 \%$ polymorphonuclear cells. No crystals were noted on polarising microscopy. A test for HIV was positive by enzyme linked immunosorbent assay (ELISA) and confirmed by Western blot. A lumbar puncture showed normal cerebrospinal fluid and a Venereal Disease Research Laboratory test was negative. His synovitis progressed and by Augus 1986 he had a symmetrical polyarthritis affecting the metacarpophalangeal joints, wrists elbows, shoulders, knees, ankles, and metatarsophalangeal joints.

He had pain at night and early morning tiffness, which lasted more than two hours. He noted profound fatigue and a weight loss of 5-7 kg with weakness, particularly of the quadriceps muscles.

The erythrocyte sedimentation rate was 135 $\mathrm{mm} / \mathrm{h}$ (Westergren)

In October 1986 a biopsy of a solitary lesion on the right thigh showed Kaposi's sarcoma, and a diagnosis of AIDS was made. Retrospectively the lesion had probably been present for 10 months before biopsy.

Laboratory data shortly after included a T4 cell count of $11 \%$ (normal 39-63), T8 cells $69 \%$ (normal 16-38), and a T4/T8 ratio of $0 \cdot 16$.

Despite treatment with zidovudine, doxorubicin, and other drugs his Kaposi's sarcoma spread to the skin and bowel. He had two episodes of shigella diarrhoea, both treated with ampicillin, with no residua and no flare of his arthritis or other change in his clinical state. Over the next 20 months his major complaint was polysynovitis and pain, which was poorly controlled with indomethacin and prednisone $10-20 \mathrm{mg}$ a day. Radiographs of the hands showed periarticular osteoporosis and erosions consistent with rheumatoid arthritis. The feet also showed osteoporosis, erosions, and marked deformity at the metatarsophalangeal joints. Spinal and pelvic radiographs were normal. A synovial biopsy was performed in September 1988 but no HIV DNA was detected by polymerase chain reaction and viral cultures were negative.

Shortly thereafter he died from a massive gastrointestinal bleed secondary to his Kaposi's sarcoma.

Winchester et al described a group of patients with AIDS with multiple criteria for Reiter's syndrome. ${ }^{2}$ The arthritis in these patients and others subsequently reported ${ }^{1}$ was predominantly oligoarticular, and a few patients had sacroiliitis. Other clinical features of the syndrome were commonly present; particularly noted were heel pain and a variety of enthesopathies. Cutaneous features were common.

A subacute oligoarthritis syndrome, possibly specific for AIDS, has been reported. A more extensive report of the rheumatic manifestations of $\mathrm{HIV}^{1}$ also recorded Reiter's arthritis, a painful articular syndrome, and psoriatic arthritis. One patient in that series was reported to have a polyarthritis of six months' duration. The presentation was that of symmetrical disease affecting small joints, which was latex negative and clinically similar to rheumatoid arthritis. No mention was made of radiographic findings or other diagnostic features.

Autoimmune disease occurs in association with AIDS. Sjögren's syndrome manifest mainly by sicca syndrome has been well reported. ${ }^{3}$ Polymyositis and vasculitis have also been described. ${ }^{4}$ Rheumatoid arthritis, seronegative or seropositive, has not been reported to be associated with HIV infection. Conversely, it has been stated that it does not occur. The polyarthritis seen has been thought to represent Reiter's arthritis or reactive arthritis.

The patient presented here fulfils criteria for classical seronegative rheumatoid arthritis. He had no other features of Reiter's syndrome or psoriasis and no enthesopathy or heel pain. The disease was prolonged with an unremitting course for about 30 months until his death. He did not respond to treatment with auranofin, penicillamine, or cytotoxic drugs and zidovudine used to treat his Kaposi's sarcoma. His disease was moderately controlled with indomethacin $75 \mathrm{mg}$ twice daily and prednisolone $10-25 \mathrm{mg}$ daily. During the course of his illness treatment with prednisolone was tapered, but this resulted in a severe flare of his arthritis, though no skin rash suggestive of psoriasis.

The disease did not remit when his CD4 T lymphocyte counts were markedly reduced, suggesting that these cells were not central to the disease or that few of them were needed, or more likely a subset of disease specific $T$ lymphocytes was unaffected in this patient. It has been suggested that AIDS might cure or markedly attenuate rheumatoid arthritis, ${ }^{6}$ but the presence of HIV certainly did not reduce the severity of the synovitis in this patient.

A causative role for HIV was not established and no viral DNA was identified by poly- merase chain reaction in the cell lines obtained from his synovial biopsy specimen. Viral cultures of synovial tissue were likewise negative for HIV.

Possibly, the disease represented a reactive arthritis, but the symmetrical involvement and chronicity mitigate against this. Also it is noted that at the onset of his disease he had no obvious infection. The natural history, immunological aspects, and pathogenesis of the arthritis seen in association with HIV are poorly understood. Although rare, a polyarthritis fulfilling current criteria for rheumatoid arthritis may occur early in the disease, and HIV should probably be tested for in all high risk patients who present with a recent onset arthritis.

$$
\begin{array}{r}
\text { A M JAFFER } \\
9834 \text { Genesee Avenue } \\
\text { Suite } 400 \\
\text { La } 7 \text { olla } \\
\text { Califormia } 92037
\end{array}
$$

USA

1 Berman A, Espinoza L, Diaz J, et al. Rheumatic manifestations of human immunodeficiency virus infection. Am 7 Med 1988; 85: 59-64.

2 Winchester R, Bernstein D H, Fischer H D, Enlow R, Solomon G. The co-occurrence of Enlow R, Solomon G. The co-occurrence of Reiter's syndrome and acquired immuno-
deficiency. Ann Intern Med 1987; 106: 19-26.

3 Couderc L J, D'Agay M F, Danon F, Harzic M, Brocheriou C, Clauvel J P. Sicca complex and infection with human immunodeficiency virus. Arch Intern Med 1987; 147: 898-901.

4 Calabrese L H, Estes M, Yen-Lieberman, et al. Systemic vasculitis in association with human immunodeficiency virus infection. Arthritis Rheum 1989; 32: 569-76.

5 Davis P, Stein M, Latif A, Emmanuel J. HIV and polyarthritis. Lancet 1988; 335: 936.

6 Bijlsma J, Derksen R, Huber-Bruning A, Borleffs J. Does AIDS 'cure' rheumatoid arthritis? Ann Rheum Dis 1988; 47: 350-1.

\section{A case of chronic Lyme arthritis in England}

Sir: The first British case of Lyme arthritis was reported recently, ${ }^{1}$ in a patient with an acute asymmetrical polyarthritis associated with erythema chronicum migrans following a definite history of tick bite. We describe a case of chronic Lyme arthritis, hitherto unreported in the British Isles. The patient had chronic symmetrical synovitis of the knees with a history of skin rash, strongly suggestive of erythema chronicum migrans, a positive serological test for Borrelia burgdorferi but no history of tick or insect bite. Her case is described below.

A 42 year old woman noticed gradual onset of pain, swelling, and stiffness in her knee joints in February 1986. In March 1987 she was seen in our rheumatology clinic, and examination showed effusions from both knees with no evidence of active inflammation. There was no cardiac or neurological abnormality and she was otherwise well. She had no personal or family history of arthritis, psoriasis, uveitis, bowel disturbances, or urogenital symptoms. She had, however, seen her general practitioner in October 1985 with an annular erythematosus rash on her trunk associated with malaise and fever. The rash had started with a reddish macula, which gradually spread to form a plaque-like rounded lesion with some raised margin. The rash was itchy and disturbed her sleep occasionally. Her general practitioner, believing it to be a non-specific skin infection, treated her with doxycycline as 
she was allergic to penicillin. Her general symptoms resolved with the antibiotic treatment and at that time there was no history of joint pain or of any insect or tick bite.

Investigations in the rheumatology clinic showed a normal haemoglobin, white cell count, and erythrocyte sedimentation rate. All biochemical tests and serological tests for rheumatoid factor, antinuclear factor, and $\mathrm{C}$ reactive protein were normal. Serological tests for syphilis were also negative. Radiological investigations of her hands, feet, knees, and sacroiliac joints were normal. Chest radiograph was normal.

About $30 \mathrm{ml}$ synovial fluid was aspirated from each knee joint in the clinic, but neither knee was injected with intra-articular steroid or local anaesthetic. Microscopy of synovial fluid from the knees showed 136 red blood cells $/ \mathrm{ml}$ and 70 white blood cells $/ \mathrm{ml}$, of which $85 \%$ were lymphocytes and $15 \%$ were polymorphs. The synovial fluid, ordinary bacteriological culture, and culture for mycobacteria were negative. Serum titre of IgG antibody to Borrelia burgdorferi was 1/256 (considered significant), and IgM antibody was not detected. The titre for Borrelia burgdorferi was confirmed on several occasions, on different blood samples.

A non-steroidal anti-inflammatory drug was prescribed and the patient responded well. This case has been reviewed in the clinic several times since; effusions in the knee joints have recurred but there has been no sign of acute inflammation. Although there was clinical evidence of chronic synovial proliferation, radiographs of the knees, repeated on several occasions, did not show any destructive lesions. She admitted to no functional difficulty, though had occasional stiffness in her knees. The synovial fluid was analysed on several occasions and cellular characteristics remained unaltered. Although polymorphonuclear leucocytosis is usually the predominant feature of 'Lyme arthritis' effusion, our patient had predominant lymphocytosis. Borrelia burgdorferi was not grown on appropriate culture. Her serum titre of IgG antibody to Borrelia burgdorferi had however dropped to 1/64 and IgM antibody was not detected in the subsequent serological tests.

Arthritis has been reported in several series as the commonest complication, occurring in about $60 \%$ of cases. ${ }^{2}$ Most patients present with an acute oligoarthritis or polyarthritis, affecting predominantly the large joints. A chronic arthritis is much less common, and only 10 cases have so far been reported from the United States, though in 1987 six more cases of chronic Lyme arthritis were recognised. ${ }^{3}$ In that series chronic arthritis was defined as persistent pain and swelling affecting at least one large joint for more than a year.

Chronic seronegative oligoarthritis affecting mainly large joints is a common diagnostic problem. Many such patients cannot be satisfactorily categorised clinically, serologically, or radiologically into one of the recognised groups of seronegative spondarthritides, such as ankylosing spondylitis, gut associated and sexually acquired reactive arthritis, psoriatic arthritis, or even seronegative rheumatoid arthritis. A proportion of these undefined cases may eventually come to be recognised as Lyme arthritis. It is clearly important to elicit a previous unrecognised history of skin rash, insect or tick bite, neurological illness, or exposure to a possible vector for Borrelia burgdorferi. Our patient lived in Waddesdon, near Aylesbury, close to a woodland area frequented by small deer, but there has been no record of positive serological tests for Borrelia burgdorferi in the general population of that area (Gillett, unpublished data).

'Early onset'-type arthritis probably has an infectious cause as Borrelia burgdorferi has been isolated from the synovial fluid ${ }^{4}$ and it is claimed that patients respond well to treatment with antibiotics such as penicillin or tetracycline, which may, in fact, prevent the appearance of late complications such as arthritis. ${ }^{56}$ In contrast, however, our patient did indeed develop chronic synovitis of the knees for more than a year, though she was treated with an appropriate antibiotic for more than seven days during the acute illness. A positive serological test for Borrelia burgdorfer is one of the most important tools of early diagnosis, though in one series only $62 \%$ had a positive test. ${ }^{7}$ This serological test has several shortcomings as it lacks specificity and interlaboratory standardisation. It is suggested that the western immunoblotting technique offers better sensitivity and specificity than indirect immunofluorescence or enzyme linked immunosorbent assay (ELISA) and may well become the standard test for Lyme disease.

It is important to realise that treatment with antibiotics may abort the development of positive antibody titres to a significant degree. Therefore the diagnosis is apt to be clinical rather than serological, and studies based solely on positive serological tests are likely to underestimate the incidence. Pathologically chronic Lyme arthropathy resembles rheumatoid arthritis, though they have distant clinical and immunogenetic characteristics. Chronic Lyme arthritis, like rheumatoid arthritis, can be destructive, and about $10 \%$ of cases develop severe erosive disease; this is often associated with HLA-DR2. Our patient, however, had no evidence of erosive disease. The pathogenesis of chronic Lyme arthritis is still far from clear, though it is believed that in genetically susceptible subjects a tick transmitted infection triggers an inappropriate immune response that may eventually localise and persist in joints, with pathological consequences.

Management of Lyme arthritis depends on the pattern of arthritis at presentation. The acute variety may well respond to antibiotic treatment with or without non-steroidal antiinflammatory drugs, but the role of intraarticular steroid during the acute phase is a matter of much debate. It is suggested that intra-articular steroids are best avoided as they may be associated with an increased incidence of antibiotic failure. ${ }^{8}$ The symptoms of chronic Lyme arthritis are treated with non-steroidal anti-inflammatory drugs with or without intra-articular steroids, as it is believed to have an immunological origin. Many patients, however, may need a surgical synovectomy because of poor response to medical treatment. Perhaps early surgical intervention in these groups of patients prevents the onset of erosive and destructive arthropathy.

We wish to thank Dr Paul Gillett and Dr David J Wright for carrying out the serological tests, and Miss Anne Dudley Smith for typing and secretarial assistance.

K K CHAKRAVARTY $M$ WEBLEY G D SUMMERS

Department of Rheumatolog Department of Rheumatolog

Oxford Regional Rheumatic Diseases Research Centre
Stoke Mandeville Hospital
Aylesbury, Buckinghamshire HP21 8AL

1 McCallan D C, Hughes C A, Bradlow A. Lyme arthritis in southern England. BMF 1987; 294 1062-3.

2 Steere A C, Bartenhagen N H, Craft J E. Clinica manifestations of Lyme disease. Zentralblatt fur Bakteriologie, Mikrobiologie und Hygiene 1986, 263: $210-5$

3 Steere A C, Schoen R T, Taylor E. The clinica evolution of Lyme arthritis. Ann Intern Med 1987; 107: 725-31.

4 Lastavica C C, Syndman D R, Schenkein D P, Berardi V P, Pariser K M. Demonstration of Borrelia burgdorferi in a patient with chronic Lyme arthritis. Zentralblatt fur Bakteriologie, Myme arthritis. Zentralblatt fur Bakteriologie,

5 Steere A C, Malawista S E, Newman J H, Spiele P N, Bartenhagen N H. Antibiotic therapy in P , Bartenhagen $N \mathrm{H}$. Antibiotic therapy in Lyme disease. Ann Intern Med 1980; 93: 1-8.

6 Steere A C, Hutchinson G J, Rahn D W Treatment of early manifestations of Lyme disease. Ann Intern Med 1983; 99: 22-6.

7 Thambidurae R M, Grigor J K, Horman J T Lyme disease: Epidermological characteristics in Maryland, 1984-86. Maryland State Medical foumal 1988; 37: 465-7.

8 Sigal L H, Steere A C Freeman D H. Proliferative responses of mononuclear cells in Lyme disease. Arthritis Rheum 1986; 29: 761-9. 